\title{
Introduction to the Special Issue: Legislation Related to Children's Evidence-Based Practice
}

\author{
Eric Trupin $^{1} \cdot$ Suzanne Kerns ${ }^{1}$
}

Published online: 16 June 2015

(c) Springer Science+Business Media New York 2015

This Special Issue addresses the passage of a law (Washington State 2011) during the 2012 legislative session and the subsequent activities that were developed as an outcome of that legislation in the State of Washington. The law requires policy makers to utilize empirically supported services in publicly funded children's behavioral health, juvenile justice and child welfare. The implications of this type of legislation nationally as a model for implementing a more empirically informed provision of children's services are significant.

There is precedent for bills such as this. For example, in 2003 Oregon passed Senate Bill 267 ("Evidence-based practice") (Oregon State 2003), which mandated increase the use of evidence- and research-based psychosocial interventions within public mental health systems (including addiction), corrections, and child welfare. Setting benchmarks across three biennia, the ultimate goal by the third and final biennium (2009-2011) was that $75 \%$ of services were required to be evidence-based.

The articles in this issue focus on the process and products that flowed out of the Washington law. Many of these processes and products were developed jointly between the University of Washington's Evidence-Based Practices Institute (EBPI) and the legislatively-funded Washington State Institute of Public Policy (WSIPP). For historical context, previous legislative funding established a program within the University of Washington to train mental health providers to choose careers in the public sector provide continuing educational opportunities to

Eric Trupin

trupin@uw.edu

1 Department of Psychiatry and Behavioral Science, Division of Public Behavioral Health and Justice Policy, University of Washington School of Medicine, Seattle, WA, USA mental health professionals and be a resource to the executive and legislative branch on issues related to behavioral health. This program was the basis for development of the Division of Public Behavioral Health and Justice Policy, housed within the Department of Psychiatry and Behavioral Sciences, within the School of Medicine. In 2007 legislation (Washington State 2007) further established a university based Evidence-Based Practice Institute (EBPI) within the Division of Public Behavioral Health and Justice Policy. The primary mission of the EBPI was to enhance the uptake of evidence-based practices in children's mental health services through expanded implementation and workforce training. Despite the existence of this Institute, no mandate, incentive or consequence existed for the child serving systems to implement evidence-based practices. The system change process was seen as moving too slowly by legislative leaders and enthusiasm built for creating a more directive approach to system change related to evidence-based practices. These factors contributed to the legislature crafting HB 2536 as a remedy to increase the speed in which the child serving systems would move in the direction of funding empirically supported interventions. In an environment of strong political partisanship it is instructive to note that this legislation was passed with a close to a unanimous vote in both the House and Senate of the Washington State legislature and was subsequently signed by the Governor.

\section{What were the Factors that Allowed for this Empirically Driven Legislation to Prosper?}

Many years of relationship building between the university's faculty and the legislature and executive branch enhanced and deepened the role of the behavioral health 
faculty and established the context for consistent faculty access and input to legislators and policy makers on issues related to social and health care services and the needs to train a workforce to support effective programs.

Two relatively unique factors contributed to the enhanced role of the University in policy access and influence. The first is the University of Washington School of Medicine currently being the only medical school and the major health sciences institution in Washington, Wyoming, Alaska, Montana and Idaho (WWAMI). The WWAMI program, which was established in the early 1970s (with Wyoming joining in 1996) provides the majority of health care training for the five participating states. A consequence of this training role was increased utilization of faculty for assistance in health care program development and policy. Both the university and policy makers were eager to support the design of training programs that aligned with the needs of the populations that resided in these states. As behavioral health, child welfare and juvenile justice issues were recognized for being closely interrelated, more interest was generated in looking for effective cross system strategies to improve outcomes for these "system" involved children and families.

The second factor was the legislature establishing in 1983 the Washington Institute for Public Policy (WISPP). WISPP's mission is to conduct practical, non-partisan research at the direction of the legislature on policy issues and proposed legislation. WSIPP is highly regarded by the Washington States legislature as well as policy makers nationally and internationally for its careful analysis of both program effectiveness and the economic and financial benefits or liabilities related to policies and programs. In 1998, WSIPP published a report entitled "Watching the Bottom Line: Cost-Effective Interventions for Reducing Crime in Washington" (Aos et al. 1998). The consequence of this report was a groundswell of legislative interest in evidence-based practices in preventing youth placement in secure juvenile residential institutions. The report demonstrated that implementing identified programs with fidelity leads to positive reductions in criminal recidivism, large effect sizes for treatment, and economic benefit. On the basis of this report the Washington legislature decided to lower their allocations for building secure placements in both the juvenile and adult correction system, diverting a portion of the resource to implementing cost-effective evidence-based practices. Significant bi-partisan interest (and multiple WSIPP reports and meta-analyses related to effective practices in behavioral health, juvenile justice, child welfare and education) was generated from that point forward in implementing evidence-based practice throughout child serving systems.

\section{What Strategies and Steps did We take to Position Ourselves to Influence Policy?}

Twenty-five years ago a paper was published with the title "Maximizing the role of state legislatures in the development of mental health policy" (Trupin et al. 1989). The logic that drove that premise was that clinician/scientists had an obligation to do more than produce quality research on effective practice. Translating evidence of effective practice into policy was an essential next step. Not an easy road to hoe for academics used to the challenges of conducting research but unfamiliar or uncomfortable functioning in policy and political environments where data were often overlooked or mistrusted. As we indicated then, "there is little empirical knowledge about the pattern of and rationale for state mental health policy decisions. Formal policy analysis and program evaluations have little to do with the true decision-making process and frequently are overshadowed by serendipitous variables." In retrospect that statement may have been a bit harsh but not incompatible with most legislative and policy experiences. The "variables" that historically impacted policy were often derived from perceptions on how well a mental health agency was being managed -frequently driven by media attention or partisan politics. In addition, legislator's opinions evolved from their own negative experiences with services and or the stigma they attached to those in need of mental health care. Subsequent to the publication of this article we have had two decades where we have endeavored to follow the following four steps to influence policy decisions, which remain relevant today:

1. Understand the state legislative process Essentially become familiar with how legislation is enacted. We suggest that in order to have impact one needs to understand the "manifest" and "latent" aspects of the legislative process. Understanding potential levers of change and opportunities and contingencies within the legislative setting is akin to understanding family dynamics. Using motivational strategies to promote change is essential to elicit engagement and new perspective taking.

2. Locate the sources of power in state government Academics motivated to bring empirical findings into the legislative arena are encouraged to participate in the political process-get to know your local legislators and the issues they are interested in. The benefits of having legislators and their aides accessible outside of legislative sessions provides many opportunities to develop relationships and alliances that can impact policy.

3. Target sources of interest Legislators may have family members or friends where the issues related to 
behavioral health are either a personal or professional interest. Providing research evidence from academics supporting effective policy or programs can have a significant impact on the moving policy in an empirically supported direction.

4. Develop and sustain consensus Conflicting perspectives typically lead to legislative inertia. Establishing a coherent "voice" related to policy direction increases the likelihood of legislative action. Academics partnering with family advocates and youth are a powerful force in supporting "real life credibility" of proposed strategies.

Although the term "implementation science" had not been utilized when we proposed the steps above, they represent a pragmatic precursor to that concept. An extensive literature had already been published on legislative politics and we have seen, in the past 15 years, rapid increases in legislator's and policy maker's interest and enthusiasm in implementing evidence-based interventions for children and youth who experience a wide range of behavioral health disorders. With the publication of various "lists" that propose to document "what works" and the expectations embedded in the Affordable Care Act related to incentivizing effective outcomes pressure has intensified in both the public and private sectors to utilize resources to support identified programs. Both nationally and internationally these decision makers have recognized and become aware of the beneficial clinical and economic effects that are attributed to these programs when they are implemented in an adherent manner with fidelity to the treatment model.

The obligations related to system and practice change expectations set into law by House Bill 2536 are far reaching in terms of public impact. The legislation however was not clear on establishing incentives for compliance or consequences for lack of compliance with the mandate to increase the use of empirically supported practices (evidence- and research-based practices and promising practices). The motivation for compliance at the State level was driven both by a sense of potential future legislative consequences as well as the leadership of the Department of Social and Health Services (DSHS) noting alignment with the goals of utilizing more effective clinical and economic practices. Despite being in the same umbrella agency (DSHS) the mental health, juvenile justice and child welfare divisions had limited experience working together in a systematic manner in planning around services. They had even less experience around orchestrating a coordinated approach to the implementation of evidence based practices. Expectations around evaluation of adherence and fidelity and the assessment of the outcomes of interventions were in either in a "pre-contemplative" stage or were addressed through the use of gross measures of treatment completion. Our strategies to move the "system" into a more action oriented direction are described in the articles in this special issue. It is clear that future policy initiatives will need to be developed that address contingencies for implementing empirically supported practices in alignment with fidelity measures and how fidelity impacts outcomes. In order to sustain provider agencies contracted to serve children continued shift to empirically supported practices, the child serving divisions in DSHS will need to develop financial incentives to make and sustain this extraordinary culture shift towards effective practices. Moving the child serving system in the direction of financial incentives for adherence and fidelity and positive outcomes is a priority for our current consultative effort. The provider agencies support for the notion of incentives for fidelity is strong but their enthusiasm for an incentive-based approach contingent on positive outcomes is significantly less enthusiastic.

The improvement of our State's economy and increased legislative assertiveness around implementing empirically supported practices bodes well for continued movement toward empirically supported approaches. The role of the University as both a "cheerleader" and broker for empirically supported practices in the community, with policy makers and the legislature, while providing oversight of adherence and fidelity, is fraught with potential concerns around conflict of interest. So far, we have navigated that issue successfully. Once contingencies are raised and incentives are provided for empirically supported practices implementation our relationships with the providers may require more careful orchestration around maintaining positive affiliations.

HB 2536 does direct (absent specific contingencies) public funds to be directed to evidence-based and researchbased practices that demonstrate efficacy in terms of the heterogeneity (children and youth enrolled in research studies needed to align with the child population in Washington State who were of minority status-currently measured at $32 \%$ ) of the children served as well as cost effectiveness. The law required that the public child serving divisions within the state's Department of Social and Health Services and the Health Care Authority (that manages Medicaid) work with the University's EBPI and WSIPP to provide a definition of evidence-based, researchbased and promising practices as an initial step in developing an "Inventory" (see Walker and Aos, this issue). These definitions would then guide the choice of programs and where they fit in terms of evidentiary support. The Inventory would be the template of treatment practices that the child serving divisions would use to ascertain how much of their current resources were devoted to the 
practices identified. The law required that the creation of a baseline of currently utilized practices that aligned with the Inventory as well as yearly reports indicating increased use of resources directed towards the identified effective practices. Where an evidence-based or research-based practice for an identified need did not exist, the EBPI was tasked with the responsibility to support proposed promising practice development in order that they increase the likelihood they could be defined as research or evidence-based (Lyon et al., this issue). The law required regular updates of the Inventory.

In 2013, the Washington State Department of Social and Health Services published an initial baseline report detailing the extent to which evidence-based practices are available in public mental health, child welfare, and juvenile justice. Services available through children's mental health included Parent-Child Interaction Therapy, Dialectical Behavior Therapy, Cognitive Behavior Therapy for anxious children, depressed adolescents, and children with symptoms of trauma (including a service delivery structure called CBT-Plus), and full fidelity Wraparound for Youth. At the time of this report, approximately 1118 children, youth and their families received these evidence-based interventions (no cost data were available). Within child welfare, Functional Family Therapy, HomeBuilders, the Incredible Years, Multi-Dimensional Treatment Foster Care, Parent Child Interaction Therapy, and SafeCare were available. Approximately 1249 children and families received an evidence-based intervention through the child welfare system (this is an under-count because no data were available about the number of children receiving Incredible Years); the state child welfare system spent $\$ 6,371,954$. Finally, within Juvenile Justice, Aggression Replacement Training, Coordination of Services, Dialectical Behavior Therapy, Functional Family Parole, Functional Family Therapy, Family Integrated Transitions, Multi-Dimensional Treatment Foster Care, and Multisystemic Therapy were available and utilized by 4198 families; the state juvenile justice system spent $\$ 24,974,745$ on implementation of evidence-based interventions.

In 2014, a subsequent report was published. In this report, the mental health system reported on progress towards a 6 year plan that eventually will lead to $45 \%$ of services being evidence-based, in gradations of $15 \%$ per biennium, and $7.5 \%$ per year. They reported meeting the first growth benchmark, with $8.1 \%$ of services being deemed evidence-based. They also undertook two new projects to assist with planning, including a service gaps analysis (reported on in this issue by Walker and colleagues) and a comprehensive cost study (forthcoming from Kerns and colleagues). The child welfare system reported on enhancing data reporting tools and an overall increase in availability of evidence-based practices.
Specifically, they experienced a $56 \%$ increase in the number of families served. However, given challenges associated with implementation, including staff turnover and no additional training resources, they project a $20 \%$ decrease in the number of families they will be able to serve over the next fiscal year. Finally, the juvenile justice system had projected a $6 \%$ increase in services and fell just shy of this benchmark at $5 \%$. Included in this report are data from the state Health Care Authority, which administers the Medicaid benefit. This system reported 7 EBPs on which to collect data: Triple P Positive Parenting Program (Levels 2 and 3), Parent Child Interaction Therapy, Cognitive Behavioral Therapy-Plus for behavior problems, anxiety and depression, Trauma-Focused Cognitive Behavioral Therapy, Theraplay, Cognitive Behavioral Therapy, and the Strengthening Families Program.

In addition, the law placed increasing responsibility on the child serving divisions to establish unified and coordinated plans for case planning as well as monitoring procedures that assured that adherence and fidelity of evidence- and research-based practices would be maintained. The law required that training of staff in evidence-based practices and the use of public funds to implement practices be more effectively coordinated between the child welfare, juvenile justice, and behavioral health systems.

As the series of articles in this special issue will elucidate, putting into practice this legislatively driven "culture shift" provides an extraordinary laboratory for the application of the principles of implementation science in a setting where the outcomes will be transparent and the consequences for the credibility of system and service improvement as a function of implementing evidencebased significant.

\section{Articles in the Series}

The articles included in this special issue describe several critical steps and activities that were necessary to enact the legislation of $\mathrm{HB} 2536$ and be responsive to community concerns. In addition to providing information about strategies employed in Washington State, each of the four articles provides tangible ideas and suggestions for other jurisdictions considering undertaking similar efforts.

As mentioned above, an initial step was to provide a definition of "evidence- and research-based." In the first article, Walker et al. describe the process of adapting and expanding current definitions for evidence-based, researchbased, and promising practices to better reflect the current state of the science (Walker et al. 2015). They describe the different existing indexes and definitions of evidencebased practices and compare them to the Washington State Inventory. The Washington State definition of 
'evidence-based' contains two requirements that extend beyond traditional definitions, namely that the programs have to demonstrate a favorable cost-benefit ratio and research must be inclusive of a heterogeneous population (percent minority status at least $30 \%$, which is reflective of Washington State in general). They go on to discuss the strategies used to determine how to identify and characterize different practices and strategies for ensuring that the inventory remains up to date.

In the second article, D'Angelo et al. describe how community engagement strategies were employed to ensure broad stakeholder buy-in for implementation of the bill (D'Angelo et al. in press). They describe specific strategies to ensure stakeholder input shaped the direction of HB2536 implementation. In this paper, a potential 'road map' for other jurisdictions interested in having stakeholder inclusion in policy implementation is provided.

In the third article, Lyon et al. describe a strategy developed to review applications by community-based programs to be included on the 'promising practices' inventory (Lyon et al. 2015). In this paper, the rational for having an informed pathway for 'home grown' interventions to be considered for the inventory is described. They detail the extent to which applications are aligned with what is known about effective practice, how these programs engage in quality assurance, and the existence of culturally-specific program elements. Most proposed programs did not meet criteria for evidence- or research-based, indicating that the strategy for identifying local practices was effective in finding high-quality programs that may not yet have the opportunity to be evaluated with more rigorous research methodology. The findings are considered alongside extant literature that describes various considerations about the field of evidence-based practice.

In the fourth and final article, Sedlar et al. describe a fidelity framework that can characterize practice across multiple evidence-based practices (Sedlar et al. in press). In this hands-on description of a model, the benefits at the agency level and policy level are described. Aligning the framework with the National Implementation Research Network (Fixsen et al. 2009), three core aspects of fidelity are described. Context refers to the training and organizational supports that are necessary for program implementation. Compliance refers to the extent to which providers engage in behaviors that enable fidelity evaluation (e.g., turning in videotapes). Competence refers to being able to demonstrate the skills necessary to deliver the intervention. Deficiencies in any of these areas require different responses. As evidence-based practice implementation continues to be required, having a structure to evaluate quality assurance and fidelity is vitally important. Specifically, this framework enables strategic recommendations to be made that can inform local policy.

\section{References}

Aos, S., Barnoski, R., \& Leib, R. (1998). Watching the bottom line: Cost-effective interventions for reducing crime in Washington. Olympia: Washington State Institute for Public Policy.

D'Angelo, G., Pullmann, M. D., Lyon, A. R. (in press). Community engagement strategies for implementation of a policy supporting evidence-based practices: A case study of Washington State. Administration and Policy in Mental Health and Mental Health Services Research.

E2SHB 2536. (2011). Concerning the use of evidence-based practices for the delivery of services to children and juveniles. Washington State.

Fixsen, D. L., Blase, K. A., Naoom, S. F., \& Wallace, F. (2009). Core implementation components. Research on Social Work Practice, 19, 531-540.

Lyon, A. R., Pullmann, M. D., Walker, S. C., \& D’Angelo, G. (2015). Community-sourced intervention programs: Review of submissions in response to a statewide call for "promising practices". Administration and Policy in Mental Health and Mental Health Services Research. doi:10.1007/s10488-015-0650-0 (online ahead of print).

Sedlar, G., Bruns, E. J., Walker, S. C., Kerns, S. E. U., Negrete, A., Trupin, E. (in press). Measuring fidelity to multiple evidence based practices in a statewide service improvement initiative. Administration and Policy in Mental Health and Mental Health Services Research.

Senate Bill 267. (2003). Evidence-based programs. Oregon State.

SHB 1088. (2007). Improving the delivery of children's mental health services. Washington State.

Trupin, E., McDermott, J., \& Forsyth-Stephens, A. (1989). Maximizing the role of state legislatures in the development of mental health policy. Hospital \& Community Psychiatry, 40, 637-638.

Walker, S. C., Lyon, A. R., Aos, S., \& Trupin, E. W. (2015). The consistencies and vagaries of the Washington State inventory of evidence-based practice: The definition of 'evidence based' in a policy context. Administration and Policy in Mental Health and Mental Health Services Research. doi:10.1007/s10488-0150652-y (online ahead of print). 